\title{
A Non-Randomized Direct Comparison of Cognitive- Behavioral Short- and Long-Term Treatment for Binge Eating Disorder
}

\author{
Barbara Schlup $^{\mathrm{a}}$ Andrea H. Meyer ${ }^{\mathrm{a}}$ Simone Munsch ${ }^{\mathrm{b}}$ \\ ${ }^{a}$ Department of Clinical Psychology and Psychotherapy, Faculty of Psychology, University of Basel, \\ ${ }^{\mathrm{b}}$ Department of Clinical Child and Adolescent Psychology, Institute of Psychology, University of Lausanne, Switzerland
}

\section{Keywords}

Quasi experiment - Binge eating disorder .

Treatment duration - CBT · Moderator

\section{Summary}

Background: To compare treatment outcomes of a cognitive-behavioral long-term (CBT-L) and short-term (CBT-S) treatment for binge eating disorder (BED) in a non-randomized comparison and to identify moderators of treatment outcome. Methods: 76 female patients with BED participated in the study: 40 in CBT-L and 36 in CBT-S. Outcome values were compared at the end of the active treatment phase (16 sessions for CBT-L, 8 sessions for CBT-S) and at 12-month follow-up. Results: Both treatments produced significant reductions in binge eating. At the end of active treatment, but not at the end of follow-up, effects of primary outcomes (e.g. remission from binge eating, EDE shape concern) were better for CBT-L than for CBT-S. Dropout rates were significantly higher in CBT-L $(35 \%)$ than in CBT-S (14\%). Moderator analyses revealed that treatment efficacy for rapid responders and individuals exhibiting high scores on the mixed dietary negative affect subtype differed between the CBT-L and CBT-S with respect to objective binges, restraint eating and eating concern. Conclusion: Findings suggest that CBT in general represents an effective treatment for BED, but that subgroups of patients might profit more from a prolonged treatment. Short, lessintensive CBT treatments could nevertheless be a viable option in the treatment of BED.

\section{Introduction}

Cognitive-behavioral therapy (CBT) is the most researched and the best-supported treatment for binge eating disorder (BED) $[1,2]$. Previous findings suggest that briefer, less costly interventions may be effective for at least a subset of patients with BED [2,3]. Direct comparisons of brief and more intensive treatments seem warranted, given the apparent responsiveness of BED to a wide range of treatment modalities [4]. In addition, research regarding factors influencing treatment outcome in BED is needed to develop more effective treatments [5-9]. This way, patients suited for low-intensity treatments could be more easily identified [9], thereby possibly improving the cost-effectiveness of treatment approaches for BED.

The aim of the present study was to compare the efficacy of a long-term and a short-term CBT treatment for individuals with BED. Both treatments have previously been evaluated with equivalent study designs and have proven to be efficacious for BED. Based on our previous findings of a marked reduction of binge eating within the first 8 weeks of the 16-session CBT [10] and the finding that the shortened intervention was efficacious for BED [11], we wanted to test to what degree, if at all, the 16 -week CBT outperformed the 8-week CBT with respect to BED-relevant outcomes.

A second goal of the present study was to examine potential moderators of treatment outcome. We aimed to examine whether i) rapid response to treatment $[5,7]$ and ii) high negative affect at pretreatment influenced the two CBT treatments differently.

\begin{tabular}{ll}
\hline KARGER & $\oplus$ 2010 S. Karger GmbH, Freiburg \\
Fax +497614520714 & Accessible online at: \\
Information@Karger.de & www.karger.com/ofa \\
www.karger.com &
\end{tabular}




\section{Participants and Methods}

\section{Participants}

Participants were drawn from two different samples, both originating from studies conducted at the University of Basel, Switzerland. One sample $(\mathrm{N}=40)$ included obese participants from a treatment trial evaluating the efficacy of a CBT program consisting of 16 sessions [12] that had been conducted during the period of 2001 to 2003 (referred to as CBT-L). The other sample $(\mathrm{N}=36)$ was drawn from a study evaluating the efficacy of a short-term CBT with 8 sessions in a waitlist-controlled design [11] that had been conducted during the period of December 2004 to June 2007 (referred to as CBT-S). The CBT-S included participants from both the immediate treatment and the 8-week waitlist condition, as prior analyses had shown that there were no differences between those groups. Inclusion and exclusion criteria were identical for both samples (consider Munsch et al. [10] and Schlup et al. [11] for further information). Participant characteristics and comparisons of the two samples are shown in table 1.

\section{Treatment Protocols}

\section{$C B T-L$}

The efficacy of the CBT-L was demonstrated by Munsch et al. [10]. The protocol included 16 weekly 90 -min group sessions in the active treatment phase and 5 booster sessions during the 12-month follow-up period. During these sessions, treatment contents discussed during the active treatment phase were revised, whereas no new treatment topics were introduced.

\section{$C B T-S$}

CBT-S is a shortened version of the CBT-L and has proven efficacy in a prior study [11]. There were 8 weekly 90 -min group sessions in the active treatment phase, followed by 5 group sessions identical to the CBT-L during follow-up treatment. The protocol mainly focused on reducing the core symptomatology of BED. In contrast to the longer version, psychoeducation on balanced nutrition and modification of body concept were only marginally targeted (for the content of the two treatment protocols please consider Munsch et al. [10] and Schlup et al. [11]).
Treatment groups in both samples started when 5-8 participants had been recruited. In both samples there were seven treatment groups carried through by fully qualified psychotherapists with specialized training in CBT and master's students of clinical psychology as co-therapists. Therapists and co-therapists in both studies were trained and supervised weekly by the senior researchers of the corresponding study (B.S. and S.M.).

\section{Assessments and Procedures}

Variables were assessed at pretreatment, at the end of treatment (i.e., following 16 weeks of CBT in the CBT-L sample and following 8 weeks of CBT in the CBT-S sample) and at 12-month follow-up. The number of self-reported binge episodes was assessed weekly during the active treatment phase (i.e., 8 times for the CBT-S and 16 times for the CBT-L) and at 3-, 6-, and 12-month follow-up.

\section{Diagnostic Interviews}

Screenings for current and lifetime mental disorders according to DSMIV-TR in the German language were conducted at pretreatment and included structured diagnostic interviews for mental disorders on Axis I [13] and Axis II [14].

\section{Eating Disorder Psychopathology}

BED diagnosis relied on DSM-IV-TR research criteria for BED and was confirmed with the Eating Disorder Examination Interview (EDE) [15, 16], a semi-structured interview with established reliability [17] and sensitivity to track changes in eating disorder psychopathology [16]. The EDE assesses features of BED, such as the number of objective binge episodes (OBEs) i.e. binge eating defined as consuming unusually large quantities of food with a subjective sense of loss of control, and abstainer rates ('remission'), i.e. proportion of patients not experiencing a binge during the last 28 days. In addition, the EDE comprises four subscales: dietary restraint, eating concern, weight concern and shape concern. Items are rated on a 7-point forced-choice scale (0-6), with higher scores reflecting greater severity or frequency of eating disorder psychopathology.

Table 1. Sample characteristics at baseline ${ }^{a}$

\begin{tabular}{|c|c|c|c|}
\hline & $\begin{array}{l}\text { CBT-L } \\
(n=40)\end{array}$ & $\begin{array}{l}\text { CBT-S } \\
(n=36)\end{array}$ & $\begin{array}{l}\text { Test statistics and } \\
\text { significance }\end{array}$ \\
\hline Mean age, years (SD) & $44.6(11.2)$ & $44.4(10.2)$ & $t_{74}=0.10, p=0.921$ \\
\hline Mean age at onset of disorder, years (SD) & $30.4(14.7)$ & $25.2(12.1)$ & $t_{64}=1.56, p=0.124$ \\
\hline Mean BMI, $\mathrm{kg} / \mathrm{m}^{2}(\mathrm{SD})$ & $33.2(4.3)$ & $33.2(6.9)$ & $t_{72}=-0.13, p=.988$ \\
\hline \multicolumn{4}{|l|}{ Percentage of participants } \\
\hline With high school degree & 100 & 100 & - \\
\hline With college education & 17.5 & 20.1 & $\chi_{1}^{2}=1.901, p=0.168$ \\
\hline Employed & 79.5 & 85.4 & $\chi_{1}^{2}=0.495, p=0.482$ \\
\hline Married or cohabiting with partner & 77.3 & 61.0 & $\chi_{1}^{2}=2.655, p=0.103$ \\
\hline \multicolumn{4}{|l|}{ Number (\%) of participants } \\
\hline With current comorbidity Axis I & $19(61.3 \%)$ & $12(33.3 \%)$ & $\chi^{2}{ }_{1}=0.809, p=0.368$ \\
\hline Depression & $5(11.4 \%)$ & $4(11.1 \%)$ & $\chi_{1}^{2}=0.001, p=0.972$ \\
\hline Anxiety disorders & $13(29.5 \%)$ & $10(27.8 \%)$ & $\chi^{2}{ }_{1}=0.030, p=0.862$ \\
\hline Lifetime comorbidity Axis I & $32(72.7 \%)$ & $19(52.8 \%)$ & $\chi_{1}^{2}=3.410, p=0.065$ \\
\hline Depression & $19(43.2 \%)$ & $12(33.3 \%)$ & $\chi_{1}^{2}=0.809, p=0.368$ \\
\hline Anxiety disorders & $13(29.5 \%)$ & $8(22.2 \%)$ & $\chi_{1}^{2}=0.549, p=0.459$ \\
\hline Comorbidity Axis II & $5(11.4 \%)$ & $2(5.6 \%)$ & $\chi_{1}^{2}=0.837, p=0.360$ \\
\hline
\end{tabular}

CBT-L = 16-session CBT; CBT-S = 8-session CBT.

${ }^{a}$ Current and lifetime diagnoses according to DSM-IV-TR were assessed using structured clinical interviews for Axis I [13] and Axis II [14] disorders. 
For the number of self-reported binge episodes, participants filled out a record sheet monitoring the number of 'episodes of overeating during which you felt out of control' during the past week.

\section{$B M I$}

Weight and height were measured on a Seca electronic balance scale (Seca, Vogel and Halke, Hamburg, Germany) and by a stadiometer. BMI was calculated as weight in kilograms divided by the square of height in meters $\left(\mathrm{kg} / \mathrm{m}^{2}\right)$.

\section{Suitability of Treatment}

Suitability of treatment was assessed with the session protocol of Graweet al. [18]. Participants rated item 19 ('I think another therapeutic procedure would be more suitable for me') on a 7-point Likert scale ranging from -3 (not at all) to +3 (yes, exactly). Ratings were assessed for each session during the active treatment phase and during follow-up.

\section{Statistical Analyses}

\section{Comparison of the Two Treatments}

Outcomes were remission from binge eating (based on EDE), number of OBEs (EDE), associated eating disorder psychopathology (four EDE subscales) and BMI.

The complete datasets of both samples were available for analysis, making it possible to compare the two intervention programs over the treatment course. To analyze continuously distributed outcome measures, we used linear mixed models $[12,19]$. In studies where missing values frequently occur and if the absence only depends on observed and not on unobserved measurements (a so-called missing at random or MAR pattern sensu [20]), linear mixed models have been shown to lead to more efficient and less biased results compared with complete case analyses or intent-to-treat analyses in which missing values have been imputed prior to the analysis using the last observation carried forward (LOCF) method. Also, results based on the LOCF method can be biased in either direction and thus can be interpreted as being conservative or liberal [21]. To analyze dichotomous outcome measures we applied a logistic-normal model representing a special case of a generalized linear mixed model (GLMM) in which the random effects structure is reduced to a random intercept only [22]. In cases where the predicted values of the dichotomous outcomes were close to 0 or 1 , rendering the computation of estimates problematic, we used in addition Fisher's exact test to compare treatments at each measurement point. The statistical models contained the treatment (CBT-L vs. CBT-S) as a between- and time as a withinsubjects factors. Predicted mean outcome values based on the mixed model/GLMM were compared between the two treatments at the end of treatment and end of follow-up. Effect sizes according to Cohen [23] for continuous variables, or Odds ratios for dichotomous variables, were calculated at the end of treatment and at the end of follow-up. To indicate statistical significance, an alpha level of 0.05 was chosen, unless otherwise specified. Analyses were done using the software programs R 2.5 [24] and SPSS for Windows 14 (SPPS, Chicago, IL, USA). For generalized linear mixed models we used in addition the R packages lme4 [25] and glmmML [26].

\section{Moderator Analyses}

A significant pretreatment variable $\times$ treatment (here: short vs. long) interaction indicates that the pretreatment variable is a potential moderator [27]. We also tested for the interaction pretreatment variable $\times$ treatment $\times$ time to include the temporal aspect of the study but never obtained any significant result. Therefore, we here focus on the results based on the two-way interaction. Based on the literature, the following two pretreatment variables were included into the analyses: i) Rapid response to treatment according to [7, 28], defined on the basis of the weekly self-monitoring records of binge eating. According to the definition of rapid response used by other authors [5], patients with a $65 \%$ or more decrease in binge eating within the first 4 weeks were considered rapid responders. We used linear mixed models to estimate the decrease in binge eating within the first 4 weeks as this allowed us to obtain estimates also for patients with missing values within that period. Rapid response was then used as a potential moderator of treatment outcome.

ii) Mixed dietary-negative affect subtype, assessed according to the suggestions by [29-31]. The variable in our study was derived by cluster analysis from BDI, BAI and the EDE subscale dietary restraint. Participants' scores on these variables were submitted to a K-means cluster analysis using the Quick Cluster algorithm from SPSS 14. Outcomes were the number of objective binges as well as the four EDE subscales dietary restraint, eating concern, weight concern, and shape concern.

The statistical models used were the same as for the comparison of the two treatments except that the moderator variable of interest was included here as a main effect and in all interaction terms.

\section{Results}

\section{Dropouts}

Dropout rates differed between the two samples, with significantly more dropouts in the CBT-L (14, or 35\% of all participants) than in the CBT-S (5, or $14 \%$ of all participants) between baseline and 12 -month follow-up ( $p=0.034 ; \chi^{2}$ test). In the CBT-L the majority of participants $(12$, or $30 \%$ of all participants) withdrew from treatment during the 16 weeks active treatment, in contrast to only one $(2.8 \%)$ participant during the 8 weeks active treatment of CBT-S $(\mathrm{p}=0.002)$. The reasons for dropouts included dissatisfaction with treatment $(\mathrm{n}=$ $6)$, lack of time $(n=2)$, major depression $(n=1)$ and other reasons $(n=3)$ in the CBT-L, and dissatisfaction $(n=1)$ in the CBT-S. During the 12-month follow-up period, there were no significant differences in the dropout rates between the two samples $(2$, or $5 \%$, and 4 , or $11 \%$ of all participants in the CBT-L and CBT-S, respectively; $\mathrm{p}=0.32$ ).

\section{Compliance and Suitability}

Participants attended on average $13.2 \pm 2.2$ (mean \pm SE), or $82 \%$ of the 16 sessions in the CBT-L, and $6.6 \pm 1.4$, or $82 \%$ of the 8 sessions in the CBT-S. During the follow-up period of 5 booster sessions in both samples, participants in the CBT-L were present at $3.9 \pm 1.5$, or $78 \%$, and participants in the CBT-S at $3.2 \pm 1.3$, or $65 \%$ of the sessions. No significant differences in compliance were found between the CBT-L and CBT-S samples ( $\mathrm{p}=0.95$ for the active treatment phase, $\mathrm{p}=0.074$ for the follow-up phase; $\chi^{2}$ test).

At the beginning and at the end of treatment, patients in the CBT-S gave significantly better ratings of suitability of treatment $(-1.3 ;-1.7)$ than patients in the CBT-L $(-0.4 ;-0.9$; $\mathrm{p}<0.05$ for both tests), but not at the end of follow-up $(-1.5$ for CBT-L, -0.9 for CBT-S, $\mathrm{p}=0.29$ ). 
Table 2. Treatment outcome variables in the CBT-L and the CBT-S at baseline, end of treatment, and end of follow-up ${ }^{\mathrm{a}}$

\begin{tabular}{|c|c|c|c|c|c|c|c|c|c|}
\hline & \multicolumn{3}{|l|}{ Baseline } & \multicolumn{3}{|c|}{ End of treatment } & \multicolumn{3}{|c|}{ End of follow-up } \\
\hline & CBT-L & CBT-S & & CBT-L & CBT-S & & CBT-L & CBT-S & \\
\hline Remission,\% (EDE) & $\begin{array}{l}0 \\
(\mathrm{n}=39)\end{array}$ & $\begin{array}{l}0 \\
(\mathrm{n}=30)\end{array}$ & - & $\begin{array}{l}86 \\
(\mathrm{n}=24)\end{array}$ & $\begin{array}{l}46 \\
(n=27)\end{array}$ & $\begin{array}{l}\mathrm{p}=0.008 \\
\mathrm{OR}=7.4\end{array}$ & $\begin{array}{l}>99 \\
(\mathrm{n}=15)\end{array}$ & $\begin{array}{l}64 \\
(n=20)\end{array}$ & $\begin{array}{l}\mathrm{p}=0.16 \\
\mathrm{OR}=127.4\end{array}$ \\
\hline $\begin{array}{l}\text { Objective binge episodes } \\
\text { (EDE) }\end{array}$ & $\begin{array}{l}14.80 \\
(n=39)\end{array}$ & $\begin{array}{l}14.80 \\
(\mathrm{n}=30)\end{array}$ & $\begin{array}{l}p=0.98 \\
E S=0.004\end{array}$ & $\begin{array}{l}0.43 \\
(\mathrm{n}=24)\end{array}$ & $\begin{array}{l}2.36 \\
(n=27)\end{array}$ & $\begin{array}{l}\mathrm{p}=0.23 \\
\mathrm{ES}=0.29\end{array}$ & $\begin{array}{l}0.01 \\
(\mathrm{n}=15)\end{array}$ & $\begin{array}{l}1.91 \\
(n=20)\end{array}$ & $\begin{array}{l}\mathrm{p}=0.43 \\
\mathrm{ES}=0.16\end{array}$ \\
\hline Dietary Restraint (EDE) & $\begin{array}{l}2.19 \\
(n=40)\end{array}$ & $\begin{array}{l}2.08 \\
(n=36)\end{array}$ & $\begin{array}{l}\mathrm{p}=0.70 \\
\mathrm{ES}=0.06\end{array}$ & $\begin{array}{l}1.01 \\
(\mathrm{n}=24)\end{array}$ & $\begin{array}{l}1.62 \\
(n=33)\end{array}$ & $\begin{array}{l}\mathrm{p}=0.06 \\
\mathrm{ES}=0.30\end{array}$ & $\begin{array}{l}1.05 \\
(\mathrm{n}=15)\end{array}$ & $\begin{array}{l}1.75 \\
(\mathrm{n}=28)\end{array}$ & $\begin{array}{l}\mathrm{p}=0.07 \\
\mathrm{ES}=0.29\end{array}$ \\
\hline Shape concern (EDE) & $\begin{array}{l}3.95 \\
(n=40)\end{array}$ & $\begin{array}{l}3.51 \\
(\mathrm{n}=36)\end{array}$ & $\begin{array}{l}\mathrm{p}=0.16 \\
\mathrm{ES}=0.25\end{array}$ & $\begin{array}{l}2.15 \\
(n=24)\end{array}$ & $\begin{array}{l}3.00 \\
(n=33)\end{array}$ & $\begin{array}{l}\mathrm{p}=0.02 \\
\mathrm{ES}=0.39\end{array}$ & $\begin{array}{l}1.91 \\
(\mathrm{n}=15)\end{array}$ & $\begin{array}{l}2.58 \\
(n=28)\end{array}$ & $\begin{array}{l}\mathrm{p}=0.10 \\
\mathrm{ES}=0.25\end{array}$ \\
\hline Weight concern (EDE) & $\begin{array}{l}3.64 \\
(n=40)\end{array}$ & $\begin{array}{l}3.39 \\
(n=36)\end{array}$ & $\begin{array}{l}\mathrm{p}=0.39 \\
\mathrm{ES}=0.14\end{array}$ & $\begin{array}{l}2.14 \\
(n=24)\end{array}$ & $\begin{array}{l}2.52 \\
(\mathrm{n}=33)\end{array}$ & $\begin{array}{l}\mathrm{p}=0.27 \\
\mathrm{ES}=0.17\end{array}$ & $\begin{array}{l}1.66 \\
(n=15)\end{array}$ & $\begin{array}{l}2.26 \\
(n=28)\end{array}$ & $\begin{array}{l}\mathrm{p}=0.14 \\
\mathrm{ES}=0.23\end{array}$ \\
\hline Eating concern (EDE) & $\begin{array}{l}1.91 \\
(n=40)\end{array}$ & $\begin{array}{l}1.73 \\
(n=36)\end{array}$ & $\begin{array}{l}\mathrm{p}=0.44 \\
\mathrm{ES}=0.13\end{array}$ & $\begin{array}{l}0.34 \\
(n=24)\end{array}$ & $\begin{array}{l}0.82 \\
(n=33)\end{array}$ & $\begin{array}{l}\mathrm{p}=0.07 \\
\mathrm{ES}=0.29\end{array}$ & $\begin{array}{l}0.16 \\
(n=15)\end{array}$ & $\begin{array}{l}0.68 \\
(n=28)\end{array}$ & $\begin{array}{l}\mathrm{p}=0.09 \\
\mathrm{ES}=0.26\end{array}$ \\
\hline BMI & $\begin{array}{l}33.14 \\
(n=40)\end{array}$ & $\begin{array}{l}33.36 \\
(n=36)\end{array}$ & $\begin{array}{l}\mathrm{p}=0.87 \\
\mathrm{ES}=0.04\end{array}$ & $\begin{array}{l}33.13 \\
(n=28)\end{array}$ & $\begin{array}{l}33.15 \\
(n=32)\end{array}$ & $\begin{array}{l}\mathrm{p}=0.98 \\
\mathrm{ES}=0.005\end{array}$ & $\begin{array}{l}32.24 \\
(n=23)\end{array}$ & $\begin{array}{l}32.63 \\
(n=27)\end{array}$ & $\begin{array}{l}\mathrm{p}=0.79 \\
\mathrm{ES}=0.06\end{array}$ \\
\hline
\end{tabular}

$\mathrm{ES}=$ Effect size according to Cohen (for continuous outcomes); OR = odds ratio (for dichotomous outcomes).

${ }^{a}$ Means denote estimates from a linear mixed model or generalized linear mixed model. Values were back-transformed if necessary.

\section{Comparison of the Two Treatments}

Table 2 provides the predicted means for all outcomes of the two treatments at baseline, end of treatment and end of follow-up.

The percentage of remission was significantly higher for CBT-L than for CBT-S at the end of treatment, but not so at the end of follow-up (based on GLMM). In contrast, Fisher's exact test performed at separate measurement points and for the available cases only gave significantly higher values for CBT-L than for CBT-S at both end of treatment $(\mathrm{p}=0.006)$ and end of follow-up ( $p=0.007)$. Treatment means for OBEs and BMI were both very similar between CBT-S and CBT-L and did not significantly differ during treatment course. With respect to eating disorder pathology, CBT-S revealed slightly better results, but significant differences between CBT-S and CBT-L were only found for EDE shape concern at the end of treatment.

\section{Moderator Analyses}

\section{Rapid Response}

$55 \%$ of all participants were classified as rapid responders. This percentage did not differ between the two treatments $\left(\chi^{2}=0.61, p=0.44\right)$. Averaged across the study period, rapid responders had lower values for OBEs in the CBT-S than non-responders (6.1 vs. 8.5), whereas in the CBT-L these two values were comparable (responders: 6.6, non-responders: 6.3; interaction rapid response $\times$ sample, $p=0.023$ ). Also respond- ers had values for the EDE subscale dietary restraint that were on average much lower than those of the non-responders (1.47 vs. 2.06) in the CBT-L but almost identical in the CBT-S (1.88 for both responders and non-responders; interaction rapid response $\times$ sample, $\mathrm{p}=0.047$ ).

\section{Mixed Dietary-Negative Affect Subtype}

Cluster analyses with the BDI, the BAI and the EDE subscale dietary restraint (EDEres) revealed two different clusters. The majority of participants $(72 \%)$ belonged to the high mixed dietary negative affect (mean values: $\mathrm{BDI}=26.7 \pm 8.1$, $\mathrm{BAI}=23.8 \pm 9.3$, EDEres $=2.31 \pm 1.21)$, and $28 \%$ to the low mixed dietary negative affect subtype (mean values: $\mathrm{BDI}=$ $9.3 \pm 5.6, \mathrm{BAI}=6.0 \pm 4.5$, EDEres $=2.06 \pm 0.99)$. Percentages in the two clusters did not differ between the two treatments $\left(\chi^{2}=0.89, p=0.35\right)$. In the CBT-S subjects of the high mixed dietary negative affect subtype had higher values for the EDE subscale eating concern than those of low subtype (1.79 vs. 0.93), whereas the corresponding values in the CBT-L were quite similar (low subtype: 1.15, high subtype: 0.98 ; interaction subtype $\times$ sample, $\mathrm{p}=0.021)$.

\section{Discussion}

This study examined a non-randomized direct comparison of a longer CBT comprising 16 sessions (CBT-L), with a shortened CBT consisting of 8 sessions (CBT-S). Both treatments were followed by booster sessions until 12-month follow-up. Overall, these findings underline the established efficacy of 
different CBT formats for BED, but not for obesity [for review see 32].

The temporal course of binge eating in both treatments was characterized by marked improvements at the beginning of treatment that were maintained until the end of follow-up. Specific comparisons of treatment outcomes at different measurement points throughout the study suggest that the CBT-L produced better outcomes than the CBT-S, especially at the end of the active treatment phase, i.e. after 8 weeks of treatment for CBT-S and after 16 weeks of treatment for CBT-L. Results of the secondary analyses thus underlined the relative superiority of the CBT-L over the CBT-S at the end of treatment and, to a lesser extent, at the end of follow-up. An important issue in this respect, however, is the significantly higher dropout rate in the CBT-L (35\%) compared to that in the CBT-S (14\%). Since there were no statistically significant differences in baseline characteristics between the CBT-L and the CBT-S (e.g. age, age of onset of disorder, $\mathrm{BMI}$, education level, or current or lifetime comorbidity rates), these factors are unlikely to influence the higher dropout rates in the CBT-L. It could be argued, however, that the dropouts may be related to the length of treatment itself, given that the reasons for dropout were dissatisfaction with treatment for six participants in CBT-L, compared to only one participant being dissatisfied with treatment in CBT-S. Slightly more than half of our study sample was classified as rapid responders. Treatment efficacy for OBEs was higher for rapid responders than non-rapid responders in CBT-S, whereas in CBT-L effects for both rapid and non-rapid responders were similar and also comparable to the effect of responders in the CBT-S. This suggests that within the shorter treatment option, patients' capacity to benefit from interventions early in treatment course is important, whereas in the CBT-L patients similarly profit from treatment until the 16th session, independent of the rapid response status. Longer treatment resulted in a better outcome in terms of EDE dietary restraint in rapid responders, whereas in CBT-S both rapid and non-rapid responders had almost identical values that were higher than those for rapid responders in CBT-L. It could therefore be hypothesized that core features of eating disorder pathology could efficaciously be improved within a prolonged time span.

The majority of the patients belonged to the mixed dietary negative affect subtype (72\%). This moderator only influenced treatment success within CBT-S and only with respect to eating concern. Subjects of high negative subtype were the least successfully treated and hence should better be treated using a prolonged treatment option. However, as we did not find any further moderating effects for other outcomes, the interpretation of this result should also take into account the possibility of chance effects.

As limitations to the present study most importantly the nonrandomized design has to be addressed. Although the two studies were conducted in very comparable settings considering specific and nonspecific factors (e.g. patient characteristics, therapist effects), only a direct, randomized comparison of the two treatments within the same setting and patients sample and conducted by the same staff could minimize the effects of uncontrolled factors (e.g. different therapists, self-selection bias because patients knew about the length of treatment). Second, the statistical power was weak because of the rather small sample size; therefore, it is possible that we were unable to detect further meaningful differences between the two treatments. Third, inferences about between-group differences are limited by the fact that many missing values occurred, particularly with respect to data at the end of follow-up.

Bearing the before mentioned limitations in mind, our findings indicate that CBT, whether short- or long-term, was an effective treatment for the majority of patients. We have demonstrated that patients classified as non-rapid responders or those exhibiting high scores on the mixed dietary negative affect subtype benefit from prolonged treatment options with respect to eating disorder pathology. Future research should re-evaluate these preliminary findings thereby taking into account long-term outcome.

\section{Disclosure}

The authors declared no conflict of interest.

\section{References}

1 National Institute for Clinical Excellence (NICE): Eating disorders - core interventions in the treatment and management of anorexia nervosa, bulimia nervosa, related eating disorders. London, NICE Clinical Guideline No. 9, 2004

2 Wilson GT, Grilo CM, Vitousek KM: Psychological treatment of eating disorders. Am Psychol 2007;62: 199-216.

3 Peterson CB, Crow SJ, Nugent S, Mitchell JE, Engbloom S, Mussell MP: Predictors of treatment outcome for binge eating disorder. Int J Eat Disord 2000;28:131-138.

7 Wilson GT, Agras WS: Practice guidelines for eating disorders. Behav Ther 2001;32:219-234.
5 Grilo CM, Masheb RM, Wilson GT: Rapid response to treatment for binge eating disorder. $\mathrm{J}$ Consult Clin Psychol 2006;74:602-613.

6 Hilbert A, Saelens BE, Stein RI, Mockus DS, Welch RR, Matt GE, Wilfley DE: Pretreatment and process predictors of outcome in interpersonal and cognitive behavioral psychotherapy for binge eating disorder. J Consult Clin Psychol 2007; 75:645-651.

7 Masheb RM, Grilo CM: Rapid response predicts treatment outcomes in binge eating disorder: implications for stepped care. J Consult Clin Psychol 2007;75:639-644.
8 Wilson GT, Fairburn CG (eds): Treatments for Eating Disorders. New York, Oxford University Press, 2002.

9 Wilson GT, Vitousek KM, Loeb KL: Stepped care treatment for eating disorders. J Consult Clin Psychol 2000;68:564-572.

10 Munsch S, Biedert E, Meyer AH, Michael T, Schlup B, Tuch A, Margraf J: A randomized comparison of cognitive behavioral therapy and behavioral weight loss treatment for overweight individuals with binge eating disorder. Int J Eat Disord 2007;40: 102-113. 
11 Schlup B, Meyer AH, Margraf J, Wilhelm F: The efficacy of a short version of a cognitive-behavioral treatment followed by booster sessions for binge eating disorder. Behav Res Ther 2009;47:628-635.

12 Munsch S, Biedert E, Meyer AH, Michael T, Schlup B, Tuch A, Margraf J: A randomized comparison of cognitive behavioral therapy and behavioral weight loss treatment for overweight individuals with binge eating disorder. Int J Eat Disord 2006; 40:1-12.

13 Margraf J: Mini-DIPS. Berlin, Springer, 1994.

14 Wittchen H, Zaudig M, Fydrich T (eds): Strukturiertes Interview für DSM-IV. Bern, Hogrefe, 1997.

15 Fairburn CG, Cooper Z (eds): The Eating Disorder Examination, 12th ed. New York, Guilford Press, 1993.

16 Hilbert A, Tuschen-Caffier B: Eating Disorder Examination: deutschsprachige Übersetzung. Münster, Verlag für Psychotherapie, 2006.

17 Grilo CM, Masheb RM, Lozano Blanco C, Barry DT: Reliability of the eating disorder examination in patients with binge eating disorder. Int $\mathrm{J}$ Ea Disord 2004;35:80-85.
18 Grawe K, Siegfried J, Bernauer F, Donati R: Quality analysis of psychotherapy research: an empirical study. Schweiz Z Psychol 1987;46:259-266.

19 Fitzgmaurice GM, Laird NM, Ware JH: Applied Longitudinal Analysis. Hoboken, Wiley, 2004.

20 Little RJA, Rubin DB: Statistical Analysis with Missing Data, 2nd ed. Hoboken, Wiley \& Sons, 2002.

21 Molenberghs G, Verbeke G: Meaningful statistical model formulations for repeated measures. Stat Sin 2004;14:989-1020.

22 Agresti A: Categorical Data Analysis, 2nd ed. Hoboken, Wiley \& Sons, 2002.

23 Cohen J: Statistical Power Analysis for the Behavioral Sciences, revised ed. New York, Academic Press, 1977.

24 R Development Core Team: R: A Language and Environment for Statistical Computing. Vienna, R Foundation for Statistical Computing, 2007, 2008.

25 Bates D, Sarkar D: Ime4: Linear mixed-effects model using s4 classes. $\mathrm{R}$ package version 0.99875 2. 2007

26 Broström G: Glmmml: Generalized linear models with clustering. $\mathrm{R}$ package version 0.65-5. 2006
27 Kraemer HC, Wilson GT, Fairburn CG, Agras WS Mediators and moderators of treatment effects in randomized clinical trials. Arch Gen Psychiatry 2002;59:877-884.

28 Grilo CM, Masheb RM: Rapid response predicts binge eating and weight loss in binge eating disorder: findings from a controlled trial of orlistat with guided self-help cognitive behavioral therapy. Behav Res Ther 2007:45:2537-2550.

3029Grilo CM, Masheb RM, Wilson GT: Subtyping binge eating disorder. J Consult Clin Psychol 2001; 69:1066-1072.

30 Loeb KL, Wilson GT, Gilbert JS, Labouvie E: Guided and unguided self-help for binge eating. Behav Res Ther 2000;38:259-272.

31 Stice E, Agras WS, Telch CF, Halmi KA, Mitchell JE, Wilson T: Subtyping binge eating-disordered women along dieting and negative affect dimensions. Int J Eat Disord 2001;30:11-27.

32 Vocks S, Tuschen-Caffier B, Pietrowsky R, Rustenbach SJ, Kersting A, Herpertz S: Metaanalysis of the effectiveness of psychological and pharmacological treatments for binge eating disorder. Int J Eat Disord 2010;43:205-217. 